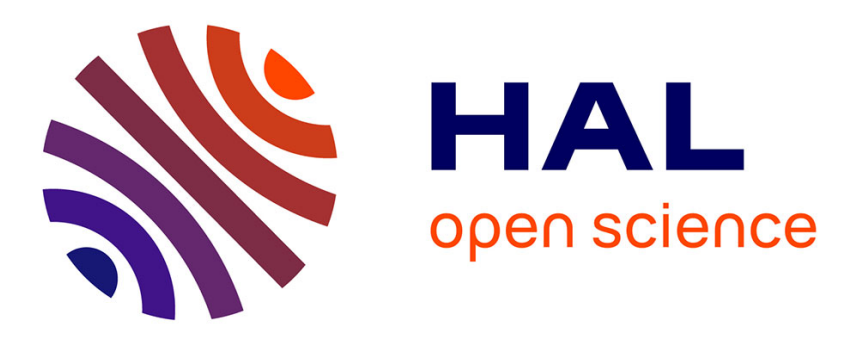

\title{
All-optical Sampling for Adaptive On-Chip Picosecond Pulse-Shaping
}

\author{
Mario Chemnitz, Bennet Fischer, Benjamin Maclellan, Piotr Roztocki, Robin \\ Helsten, Benjamin Wetzel, Brent Little, Sai Tak Chu, David Moss, Jose \\ Azana, et al.
}

\section{To cite this version:}

Mario Chemnitz, Bennet Fischer, Benjamin Maclellan, Piotr Roztocki, Robin Helsten, et al.. Alloptical Sampling for Adaptive On-Chip Picosecond Pulse-Shaping. Nonlinear Optics 2021, Aug 2021, Online, United States. hal-03418007

\section{HAL Id: hal-03418007 https://hal.science/hal-03418007}

Submitted on 17 Nov 2021

HAL is a multi-disciplinary open access archive for the deposit and dissemination of scientific research documents, whether they are published or not. The documents may come from teaching and research institutions in France or abroad, or from public or private research centers.
L'archive ouverte pluridisciplinaire HAL, est destinée au dépôt et à la diffusion de documents scientifiques de niveau recherche, publiés ou non, émanant des établissements d'enseignement et de recherche français ou étrangers, des laboratoires publics ou privés. 


\title{
All-optical Sampling for Adaptive On-Chip Picosecond Pulse-Shaping
}

\author{
Mario Chemnitz ${ }^{1}$, Bennet Fischer ${ }^{1}$, Benjamin MacLellan ${ }^{1}$, Piotr Roztocki ${ }^{1}$, Robin Helsten ${ }^{1}$, Benjamin Wetzel ${ }^{2}$, \\ Brent Little ${ }^{3}$, Sai Chu ${ }^{4}$, David Moss ${ }^{5}$, José Azaña ${ }^{1}$, and Roberto Morandotti ${ }^{1,6}$ \\ ${ }^{I}$ Institut National de la Recherche Scientifique (INRS-EMT), 1650 Boulevard Lionel-Boulet, Varennes, Quebec, J3X 1S2, Canada \\ ${ }^{2}$ XLIM Research Institute, CNRS UMR 7252, Université de Limoges, Limoges 87060, France \\ ${ }^{3} X i$ 'an Institute of Optics and Precision Mechanics, Chinese Academy of Science, Xinxi Ave, Xi'an, Shaanxi, China \\ ${ }^{4}$ City University of Hong Kong, Tat Chee Avenue, Kowloon, Hong Kong, China \\ ${ }^{5}$ Optical Sciences Centre, Swinburne University of Technology, Hawthorn, VIC 3122, Australia \\ ${ }^{6}$ Institute of Fundamental and Frontier Sciences, University of Electronic Science and Technology of China, Chengdu, Sichuan, China \\ Author e-mail address: mario.chemnitz@inrs.ca; roberto.morandotti@inrs.ca
}

\begin{abstract}
We present autonomous pulse-shaping based on on-chip temporal coherence synthesis empowered by passively detuned, nonlinear optical sampling. Our scheme enables robust, efficient and reconfigurable picosecond waveform shaping with low detection requirements and low computational power. (C) 2021 The Author(s)
\end{abstract}

\section{Introduction}

Control over the temporal shape of picosecond optical pulses is essential for many application fields, for example to boost the efficiency of all-optical telecommunication methods [1] or nonlinear light-matter interactions [2]. Most techniques process the pulse in the spectral domain, which is very powerful for femtosecond and few-picosecond pulses [3]. Unfortunately, such methods become increasingly impractical for larger pulse durations. Several systems for picosecond pulse-shaping have been developed with a recent focus on integrated approaches [4,5], as these offer smaller footprints, better energy efficiencies, and a potential for mass fabrication. However, current approaches have two main disadvantages: Firstly, they rely predominantly on a priori sample design towards a specific shaping tasks, thus lacking reconfigurability; Secondly, they do not feature optical output monitoring for easy deployment and usage. Adaptive solutions, especially ones based on machine-learning algorithms, are an attractive means to greatly increase reconfigurability [6], but are hindered by the absence of efficient and inexpensive monitoring solutions. Here, we demonstrate an on-chip system for temporal coherence synthesis [7] for 3-100 ps pulse-shaping. The backbone of our method is an all-optical sampling technique alongside a particle swarm algorithm for parameter optimization, enabling effortless reconfigurability and autonomous operation with minimal detection requirements.

\section{Results and Discussion}

Figure 1a shows the experimental implementation of our adaptive pulse-shaping device. The source is a commercial fiber laser (Pritel FFL) that emits pulses with $\sim 22$ ps FWHM (full width at half maximum) pulse duration at a repetition rate of $f_{\text {rep, },}=10 \mathrm{MHz}$. The optical chip for the pulse-shaping is based on silicon-oxi-nitride glass that offers exceptionally low optical losses [8]. The chip consists of a concatenation of multiple unbalanced MachZehnder interferometers with bit-wise increasing delays [9], as illustrated in Fig. 1a. Here, at each interferometer, the incoming pulse is split into two copies, with the splitting ratios controlled by applied heater voltages. While one copy propagates in a longer path (illustrated with different colors in Fig. 1a), the other copy takes a shorter one. Afterwards, the two parts interfere in another controllable waveguide coupler to create a new temporal waveform.

For reading out the waveform, we make use of an all-optical sampling scheme based on four-wave mixing in a highly nonlinear fiber (OFS Fitel, zero dispersion wavelength $1546 \mathrm{~nm}, 1 \mathrm{~km}$ length) [10] (see Fig. 1b). The sampling source is a spectrally filtered, mode-locked fiber laser (Menlo FC1500-250-WG) which emits pulses with 4.4 ps duration (FWHM) at a repetition rate of $f_{\text {rep }, 2}=250.27 \mathrm{MHz}$. Inside the highly nonlinear fiber, an optical idler is generated only when the pump (i.e., shaped pulse) and seed (i.e., short sampling pulse) temporally overlap. Fig. 1c shows an output spectrum of the HNLF with the generated idler (sampled output) in blue. A suitably chosen slight mismatch in the repetition rates of the pump and seed (i.e., $f_{\text {rep }, 2}=n f_{\text {rep }, 1}+\delta f$ with rate detuning $\delta f$ ) enables the passive scanning of the sampling pulse over the created waveform (pump) with each repetition of the pump pulse. Assuming a constant pulse shape, this passive method generates sampling points at kilohertz rates (i.e., with a sampling period of $\Delta T=(1 / \delta f)>1 \mu \mathrm{s})$, which are easy to filter and measure using a slow photodiode. A continuous waveform is then retrieved by using the Hilbert transform to obtain the envelope over the sampling points.

To confirm the calibration of our sampling method, we compared it against auto-correlation results. The measurements in Fig. 1d show that the retrieved envelope width from the sampling matches the pulse width measured with the auto-correlator very well. Hence, this scheme allows for unambiguous detection of the pulse 
shape in the picosecond domain with reduced experimental complexity and straight-forward low-bandwidth electronics.

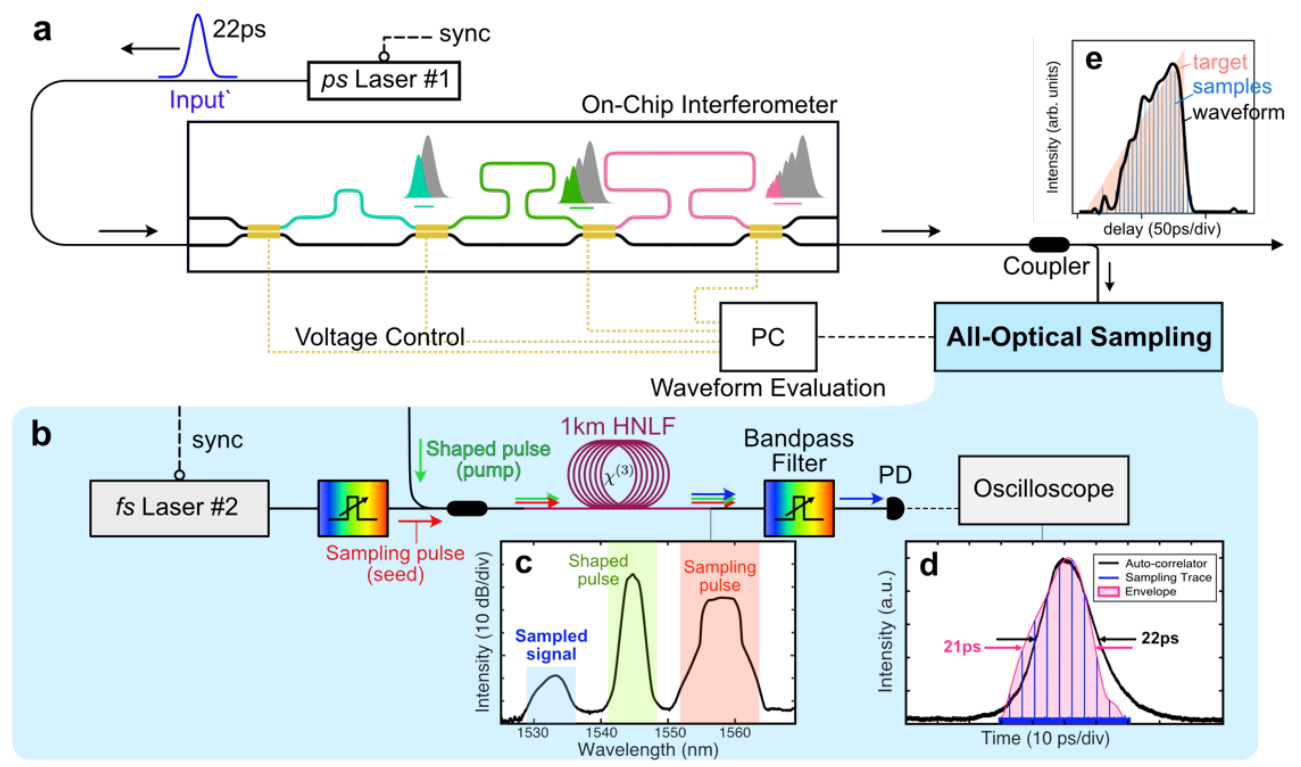

Fig. 1 a) Concept for the autonomous pulse shaper based on the concatenation of on-chip interferometers with ascending delays (in color). $\mathrm{PC}=$ personal computer, Solid lines = optical paths, dashed lines = electrical paths; b) All-optical sampling scheme. HNLF = highly nonlinear fiber, $\mathrm{PD}=$ photo diode. $\mathbf{c}$ ) Output spectrum of HNLF. d) Sampling of the 22 ps input pulse compared to its measured auto-correlation. The time scale is corrected for the respective scaling factors. e) Measured pulse shaper output (optical samples and reconstructed waveform) after optimization for a positive sawtooth shape (target).

Finally, the optical sampling was used in combination with a metaheuristic particle swarm optimization (PSO) algorithm to find the optimal coupler splitting ratios for each on-chip interferometer. For waveform evaluation in the PSO, we defined a cost function based on the cosine similarity (i.e., a measure for the similarity between two vectors, here the target and measured waveform). This way, the split-and-delay procedure could be steered such that the output pulse shape matched a given target waveform. The combination of an optimization algorithm with an efficient measurement technique provides broad reconfigurability over a vast range of targeted pulse widths, even though the platform is not specifically designed for this particular task. In our experiments, we tested and achieved standard waveforms such as negative/positive sawtooth, square and triangular shapes (e.g., see Fig. 1e). Simulation results (incl. PSO) further reveal a functional operation range from 3 to $>100$ ps target duration, with significant improvements in the waveform shape when phase control in the pulse-copy recombination is added to the system.

\section{Conclusion}

In conclusion, we have demonstrated an autonomous pulse-shaping device based on the unique combination of onchip temporal coherence synthesis, all-optical sampling, and an evolutionary optimization algorithm. The sampling technique allowed us to unambiguously distinguish between symmetric and asymmetric waveforms (e.g., sawtooth and triangle) with $20-50$ ps widths, which is particularly challenging with auto-correlators. The full scheme can easily be integrated on a photonic chip platform, using spiral waveguides for optical sampling, and hence paves the way to compact, user-friendly ps-pulse shapers for telecom applications, among others.

\section{References}

1. F. Parmigiani, et al., "Time domain add-drop multiplexing scheme enhanced using a saw-tooth pulse shaper," Opt. Express 17, 8362 (2009).

2. J. A. Fülöp, et al., "Shaping of picosecond pulses for pumping optical parametric amplification," Appl. Phys. B 87, 79-84 (2007).

3. A. M. Weiner, "Ultrafast optical pulse shaping: A tutorial review," Opt. Commun. 284, 3669-3692 (2011).

4. S. Liao, et al., "Arbitrary waveform generator and differentiator employing an integrated optical pulse shaper," Opt. Express 23, 12161 (2015).

5. S. Liao, et al., "Photonic arbitrary waveform generator based on Taylor synthesis method," Opt. Express 24, 24390 (2016).

6. G. Genty, et al., "Machine learning and applications in ultrafast photonics," Nat. Photonics 15, 91-101 (2021).

7. Y. Park, et al., "Transform-limited picosecond pulse shaping based on temporal coherence synthesization," Opt. Express 15, 9584 (2007).

8. D. J. Moss, et al., "New CMOS-compatible platforms based on silicon nitride and Hydex for nonlinear optics," Nat. Photonics 7, 597-607 (2013).

9. B. Wetzel, et al., "Customizing supercontinuum generation via on-chip adaptive temporal pulse-splitting," Nat. Commun. 9, 4884 (2018). 10. T. Dingkang, et al., "Ultrashort optical pulse monitoring using asynchronous optical sampling technique in highly nonlinear fiber," Chinese Opt. Lett. 8, 630-633 (2010). 